\title{
Phosphorylation of Connexin-43, Gap Junctional Protein, in Cumulus Cells is Regulated by Mitogen-Activated Protein Kinase and Phosphatidylinositol 3-Kinase during In Vitro Meiotic Resumption in Porcine Follicular Oocytes
}

\author{
Masayuki Shimada and Takato Terada* \\ Faculty of Applied Biological Science, Hiroshima University, Higashi-Hiroshima, \\ Hiroshima 739-8528, Japan
}

\begin{abstract}
The changes of $\mathrm{Cx}-43$ and MAP kinase in cumulus cells that were recovered from porcine cumulus oocyte complexes (COCs) cultured with FSH and LH and/or PI 3-kinase inhibitor, LY294002, were analyzed by immunoblotting. Three migration forms of $\mathrm{Cx}-43$ (43, 45 , and $47 \mathrm{kDa}$ ) were detected in the cumulus cells of COCs immediately recovered from their follicles. At 12hr of cultivation with hormones, oocyte nuclear stage was progressed from GV II stage to GV III stage. At this time point, the $43 \mathrm{kDa}$ band almost completely disappeared, although two phosphorylation forms of CX-43 (45 and 47 $k D a)$ were still detected. However, in the cumulus cells of COCs cultured with LY294002, three prominent bands of $C x-43$ were recognizable, as in the cells recovered immediately from the follicles. Although two intense bands of activated MAP kinase $(E R K 1,2)$ were also seen in the cumulus cells of COCs at 12-hr of cultivation with hormones, the intensity of the activated ERK 2 band was relatively faint in cumulus cells of COCs cultured with LY294002. At the same time point, LY294002 produced a significant decrease in the proportion of oocytes exhibiting GV III stage compared to those observed with hormones. It is concluded that the progression from GV II stage to GV III stage in porcine oocytes may be closely associated with phosphorylation of $\mathrm{CX}-43$ by virtue of MAP kinase, which was activated through the PI 3-kinase pathway in their cumulus cells.
\end{abstract}

Key words: Porcine follicular oocytes, PI 3-kinase, Connexin-43, MAP kinase, Gap junction.

Received: November 25, 1998

Accepted: December 10, 1998

*To whom correspondence should be addressed.
In most mammalian oocytes, the diplotene stage of the first meiotic prophase is a stage of arrested development in prenatal life. It is generally accepted that some component within the follicular environment surrounding an oocyte is responsible for meiotic arrest, since when oocytes are released from their follicles, they can mature spontaneously in vitro in a suitable medium [1, 2]. In follicles, cumulus cells and granulosa cells are closely attached to oocytes and synthesize some inhibitory factors, such as cyclic AMP (cAMP), hypoxanthine, and some other unknown factors, which are transported into oocytes via numerous gap junctions [3-5]. Moreover, Larsen et al. [6, 7] and Isobe et al. [8] reported that meiotic resumption in rat and pig oocytes is induced by the disruption of gap junctions within cumulus cells due to the blockage of the conduction of meiosis inhibitory signals from the outer cumulus cells to the oocytes.

Gap junctions are specialized regions in apposite membranes of neighboring cells. They are important for cellular interactions and signal transduction because they allow nonspecific transfer of low-molecular-weight molecules [9]. Gap junctions consist of hexameric structures of connexin (Cx) molecules (connexon) [9]. Itahana et al. $[10,11]$ reported that five members of connexin genes, Cx-26, Cx-30.3, Cx-32, Cx-43, and Cx-60, are expressed in porcine ovarian follicles. $\mathrm{Cx}-43$ has numerous phosphorylated sites and these phosphorylations play a key role in putative regulatory mechanisms that may operate at several levels, such as in the assembly of connexons into gap junctions in the plasma membrane and in the gating of the formed gap junction [12, 
13]. Epidermal growth factor and lysophosphatidyc acid have recently been shown to stimulate phosphorylation of serine on $\mathrm{Cx}-43$ via mitogen-activated protein kinase (MAP kinase [ERK 1,2]) and then close gap junctional communication in rat liver cells [14, 15].

In a previous experiment [16], we investigated the resumption of meiosis in porcine follicular oocytes cultured in the presence of two Phosphatidylinositol 3-kinase (PI 3-kinase) specific inhibitors (wortmannin and LY294002). The results of this experiment demonstrated that meiotic resumption of porcine follicle oocytes was blocked by the inhibition of PI 3-kinase activity within the cumulus cell itself, and it was concluded that PI 3kinase in cumulus cells may be one of the factors regulating meiotic resumption in mammalian oocytes. PI 3-kinase is one of the activators of the MAP kinase cascade, since PI 3-kinase activity is required downstream of Ras that phosphorylates c-raf, and activated c-raf is MAP kinase kinase kinase $[17,18]$. On the basis of these reports, it was deduced that $\mathrm{Cx}-43$ and MAP kinase (ERK 1,2) in cumulus cells regulated by PI 3-kinase are associated with in vitro porcine meiotic resumption. In order to gain further insights into the mechanisms that initiate meiosis in porcine oocytes, we tried to determine the nuclear morphology and phosphorylation status of $\mathrm{Cx}-43$ and MAP kinase (ERK 1,2) in the cumulus cells attached to oocytes cultured in the presence of gonadal hormones and/or PI 3-kinase inhibitor. The results show that the progression from GV II stage to GV III stage in porcine oocytes is closely associated with phosphorylation of $\mathrm{Cx}-43$ via MAP kinase activated through the PI 3-kinase pathway in cumulus cells.

\section{Materials and Methods}

\section{Isolation and culture of porcine cumulus oocyte complexes (COCs)}

Porcine ovaries were collected from prepubertal gilts at a local slaughterhouse and transported to the laboratory in $0.85 \% \mathrm{NaCl}$ containing $0.1 \mathrm{mg} / \mathrm{ml}$ kanamycin (Meiji Seika, Tokyo, Japan) at about $30^{\circ} \mathrm{C}$ within 1.5 -hr. The surfaces of follicles ranging from 3 to $8 \mathrm{~mm}$ in diameter were dissected with a razor blade and oocytes were collected by scraping the inner surface of the follicle walls using a surgical blade. The recovered oocytes were placed in prewarmed phosphate-buffered saline $(\mathrm{pH} 7.4)$ supplemented with $0.1 \%$ polyvinyl-pyrrolidone (PVP) (Sigma Chemical Co., St. Louis, USA). Oocytes with evenly granulated cytoplasm with at least 4 layers of unexpanded cumulus oophorus cells were selected under a stereomicroscope and washed 3 times with maturation medium. COCs were cultured for a given period in $100 \mu \mathrm{l}$ drops of maturation medium (about 20 oocytes/drop) covered with mineral oil (Sigma) at $39^{\circ} \mathrm{C}$ in a humidified atmosphere of $5 \% \mathrm{CO}_{2}$ in air. The basic medium was modified NCSU37 [19] containing $7 \mathrm{mM}$ Taurine (Sigma), 10\% essential and 5\% non-essential amino acids (Gibco BRL, Grand Island, NY, USA), and $0.3 \%$ PVP.

\section{Assessment of nuclear maturation}

After incubation, the oocytes were freed from cumulus cells, then mounted on slides, fixed with acetic acid/ ethanol (1:3) for at least 48-hr, and stained with acetolacmoid before examination under a phase-contrast microscope $(400 \times)$ for evaluation of their chromatin configuration. Oocytes were classified into five categories (GV I, II, III, IV, and GVBD) according to the descriptions reported by Motlik and Fulka [20].

\section{Detection of $C X-43$ and active MAP kinase (ERK 1,2) by immunoblotting analysis}

Cumulus cells recovered from COCs were solubilized on ice in a cell lysis buffer $\left(2.0 \times 10^{3}\right.$ cells $\left./ \mu \mathrm{l}\right), 20$ $\mathrm{mM}$ Tris (pH 7.5), $150 \mathrm{mM} \mathrm{NaCl}, 1 \mathrm{mM}$ EDTA, $1 \mathrm{mM}$ EGTA, 1\% Triton X-100, $2.5 \mathrm{mM}$ sodium pyrophosphate, $1 \mathrm{mM} \beta$-Glycerophosphate, $1 \mathrm{mM} \mathrm{Na} \mathrm{VO}_{4}, 1 \mu \mathrm{g} / \mathrm{ml}$ Leupeptin, and $1 \mathrm{mM}$ PMSF (Sigma). Drugs except for PMSF were purchased from New England Biolabs (Tozer Road, Beverly, MA, USA). The cumulus cell suspension was cleared by centrifugation at $15,000 \times \mathrm{g}$ for 30 $\min$ at $4^{\circ} \mathrm{C}$. After addition of an equal volume of $\times 2$ Laemli sample buffer [21], the suspension was boiled for 3 minutes and separated by SDS-PAGE on $12.5 \%$ polyacrylamide gel (Phalmacia Biotech, Uppsala, Sweden). Proteins were transferred onto a PVDF membrane (Amersham, Arlington Heights, IL, USA) using the PhastTransfer system (Phalmacia). The membrane was blocked with SuperBlock (PIERCE, Rockfold, IL, USA), and $\mathrm{Cx}-43$ and phosphorylated MAP kinase were detected with mouse anti-connexin-43 monoclonal antibody (Chemicon International INC., Temecula, CA, \#MAB3068) and rabbit anti-phospho-specific p44/42 MAP kinase monoclonal antibody (New England Biolabs) respectively, using a blotting chemiluminesence detection kit (ECL Plus, Amersham), according to the manufacture's instructions.

\section{Experimental design}

In Experiment 1, the time-dependent changes in phosphorylation of $\mathrm{Cx}-43$ in cumulus cells during meiotic resumption was examined. Selected COCs were cul- 


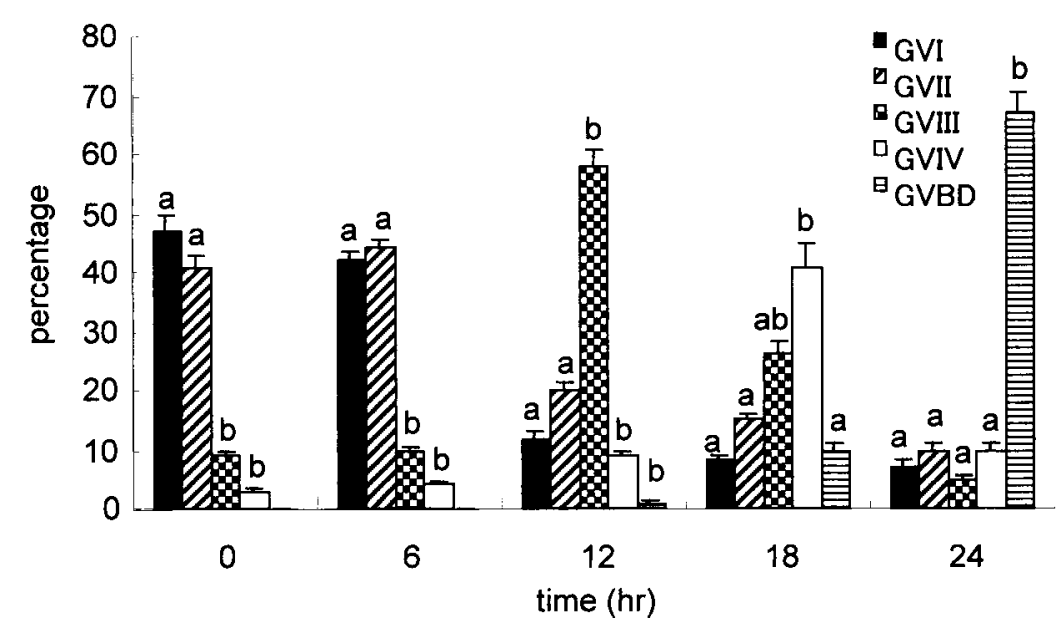

Fig. 1. Time-dependent change of nuclear maturation in the presence of FSH and LH. ${ }^{\text {a-b }}$ Defferent superscripts within columns in each group denote significant differences $(\mathrm{P}<0.01)$. GV: germinal vesicle, GVBD: germinal vesicle breakdown.

tured in a medium supplemented with $0.6 \mu \mathrm{g} / \mathrm{ml} \mathrm{pFSH}$ (Sigma) and $1.3 \mu \mathrm{g} / \mathrm{ml}$ eLH (Sigama) for $0,6,12,18$, and 24-hr. In Experiment 2, the effects of LY294002 (Sigma), a PI 3-kinase inhibitor, on phosphorylation of Cx-43 and MAP kinase (ERK 1,2) were evaluated in cumulus cells originating from $\mathrm{COCs}$ incubated until meiotic resumption was initiated. COCs were cultured for 12-hr in a medium supplemented with gonadal hormone and/or $5.0 \times 10^{-5} \mathrm{M}$ LY294002, as described in a previous report [16]. The control COCs for the collection of cumulus cells were cultured in the basic medium. Cell lysates were subjected to immunoblotting analysis.

\section{Statistical analysis}

Statistical analyses of the data (from three or four replicates for comparisons) were carried out by analysis of one-way ANOVA followed by Duncan's multiple ranges test. All percentage data were subjected to arcsine transformation before statistical analysis. Probabilities of less than 0.01 were considered to be statistically significant.

\section{Results}

\section{Experiment 1}

The time-dependent changes of the nuclear maturation are shown in Fig. 1. Before cultivation and after 6-hr cultivation, the proportions of oocytes arrested at GV I or GV II stage were significantly higher than those arrested at other stages. After a further 6-hr cultivation

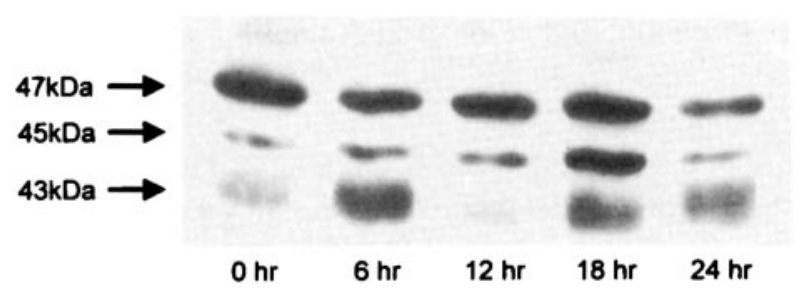

Fig. 2. Profiles in phosphorylation states of $\mathrm{Cx}-43$ in $\mathrm{cu}-$ mulus cells from porcine COCs cultured in the presence of FSH and LH.

(12-hr), marked reductions in GV I or GV II oocytes were noted and the proportion of oocytes exhibiting the GV III stage increased dramatically. When COCs had been cultured for 24-hr, oocytes that had undergone GVBD accounted for $67 \%$.

As shown in Fig. 2, three bands situated on SDSPAGE at regions, 43,45 , and $47 \mathrm{kDa}$ were detected in lysates of cumulus cells, which were isolated from COCs either immediately recovered from their follicles or cultured for 6-hr. The $43 \mathrm{kDa}$ band almost completely disappeared after 12-hr incubation, but the intensities of the $45 \mathrm{kDa}$ and $47 \mathrm{kDa}$ bands were comparable to those observed after 6-hr incubation. After a further 6-hr cultivation (18-hr), the relative intensity of the fastest migrating band was almost restored to levels observed after 6 -hr cultivation. At 18-hr of cultivation, the $45 \mathrm{kDa}$ band was much stronger than at any other time point. After 24-hr cultivation, all three bands were recognized, 


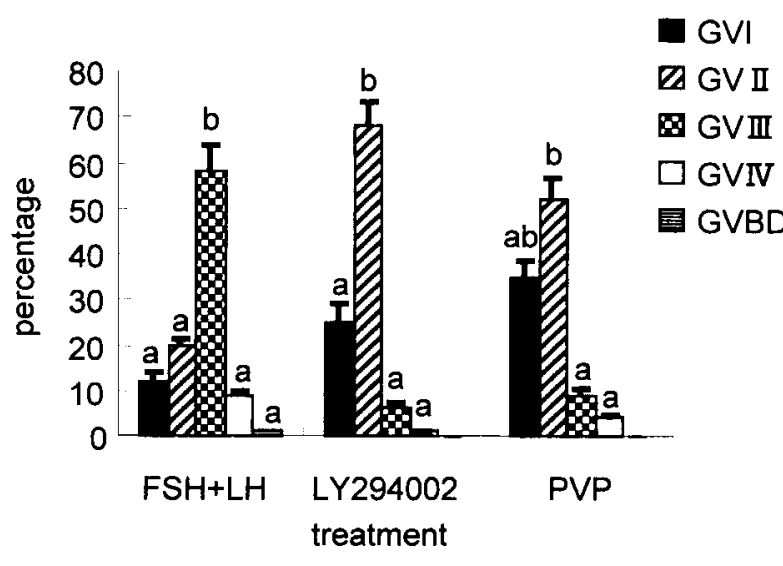

Fig. 3. Effects of LY294002 on meiotic progression in porcine COCs at 12-hr after the start of incubation. a-b Different superscripts within columns in each group denote significant differences $(\mathrm{P}<0.01)$. GV: germinal vesicle, GVBD: germinal vesicle breakdown.

but each band's intensity was much fainter than those observed at 6 and 18-hr of cultivation.

\section{Experiment 2}

Fig. 3 shows the effect of LY294002, the PI 3-kinase inhibitor, on meiotic progression in porcine cumulus oocyte complexes. The 12-hr cultivation resulted in a significantly higher proportion of oocytes arrested at the GV II stage, compared to other stages in either the presence of $50 \mu \mathrm{M}$ LY294002 or the absence of gonadal hormone. However, the GV III stage was exhibited in the majority (approximately 60\%) of oocytes cultured for 12-hr in the medium supplemented with gonadal hormones (FSH and $\mathrm{LH}$ ).

In lysates of cumulus cells isolated from porcine cOCs immediately recovered from follicles, a single prominent band of around $45 \mathrm{kDa}$ was detected by immunoblotting with anti-phospho-MAP kinase monoclonal antibody. Cultivation for 12-hr in medium supplemented with $\mathrm{FSH}$ and $\mathrm{LH}$ led to the appearance of two strong bands situated in the 43 and $45 \mathrm{kDa}$ regions. When cumulus cells were isolated from COCs which were incubated for 12-hr in the presence of LY294002, two bands, situated at 43 and $45 \mathrm{kDa}$, were observed. The intensity of the $45 \mathrm{kDa}$ band in the presence of LY294002 was comparable to that observed with FSH and LH. However, in the presence of LY294002, the $43 \mathrm{kDa}$ band was slightly fainter than it was in the presence of the hormones. Additionally, both bands derived from cumulus cells cultured in a basic culture medium (PVP) closely resembled those
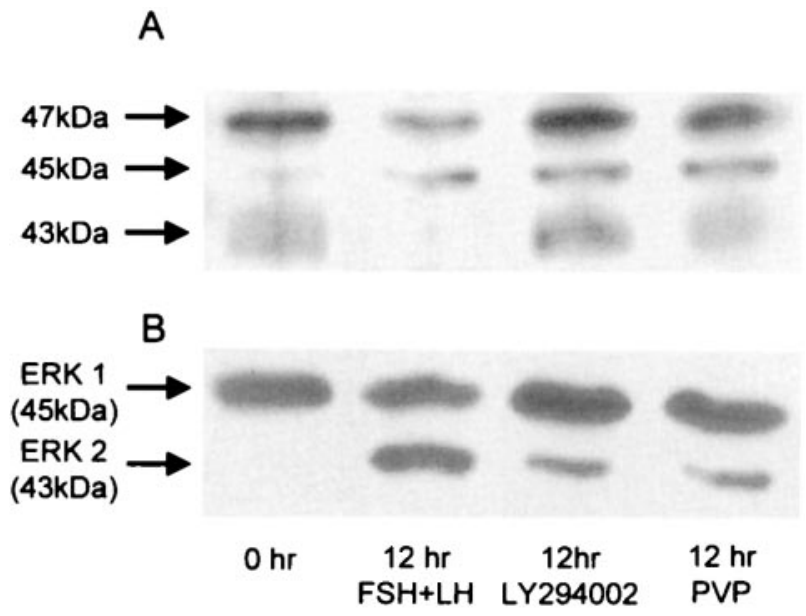

Fig. 4. Effects of LY294002 on the phosphorylation of Cx43 and MAP kinase in cumulus cells from porcine COCs. A; detection with Cx-43 antibody, B; detection with phospho-MAP kinase antibody which reacts activated from of MAP kinase.

derived in the presence of LY294002.

After 12-hr cultivation, the analysis of $\mathrm{Cx}-43$ by immunoblotting did not show any marked differences among FSH and LH-supplemented medium, LY294002supplemented medium, and a basic culture medium (PVP) in the relative intensity of slower and middle migration forms of this protein. Nonetheless, the intensity of the fastest migration form ( $43 \mathrm{kDa}$ ) almost completely disappeared in the presence of $\mathrm{FSH}$ and $\mathrm{LH}$. The intensity of the $43 \mathrm{kDa}$ band in the presence of LY294002 and in the absence of gonadal hormone was similar to that observed in cumulus cells isolated from COCs that were recovered immediately from the follicles.

\section{Discussion}

According to Lenhart et al. [22], connexin 43 (Cx-43) is expressed and phosphorylated during the estrous cycle in the ovaries of mature pigs and is phosphorylated even during follicular development when eCG and hCG are administered to prepubertal gilts. Furthermore, during in vitro cultivation of rat follicles in medium supplemented with luteinizing hormone (LH), phosphorylation of $\mathrm{Cx}-43$ in follicular cells has been reported to be induced through a PKA-dependent pathway [23]. However, the characterization and the role of $\mathrm{Cx}-43$ in in vitro meiotic reinitiation of cumulus oocyte complex have yet to be elucidated.

In agreement with some reports using mouse $L$ cells, mouse fibroblast cells, and rat epithelial cells [14, 24, 
25], the present study showed three bands on SDSPAGE situated in the 43,45 , and $47 \mathrm{kDa}$ regions. These bands were detected in the lysates of cumulus cells isolated from COCs immediately recovered from their follicles, and this is the first report that three migration forms of $\mathrm{Cx}-43$ were detected in porcine cumulus cells. Additionally, we observed in cumulus cells isolated from COCs cultured for 12-hr in the presence of $\mathrm{LH}$ and follicle stimulating hormone (FSH), that the faster migration form of $\mathrm{Cx}-43$ (43 kDa) almost completely disappeared. In Western blot analysis of mouse 3T3 fibroblasts, it has been shown that $43 \mathrm{kDa}$ is a primary translation and that either 45 or $47 \mathrm{kDa}$ is a phosphorylation form of $43 \mathrm{kDa}$ [25]. On the basis of observations that the phosphorylation of $\mathrm{Cx}-43$ leads to a closure of gap junctional communication [12], the present results strongly suggest that in porcine cumulus-oocyte complexes cultured for 12-hr in a medium with hormones, Cx-43 is phosphorylated and then gap junctional communication between cumulus cells surrounding the oocytes closes down.

The loss of cumulus-cumulus cell gap junction preceding the induction of meiotic resumption was reported in mouse COCs [26], and rat COCs [6, 7]. In pigs, removal of the cumulus cells from COCs produced a significant increase in the proportion of oocytes undergoing GVBD [5]. More recently, Isobe et al. [8] studied the regulatory mechanisms involved in meiotic resumption in pig oocytes incubated with gonadotropins. They determined the disruption time of cell-cell gap junction using a fluorescent dye (lucifer yellow). Isobe et al. [8] demonstrated that at onset and 8-hr of cultivation, the dye was transferred into all cumulus cells surrounding oocytes. However, at 16-hr cultivation, the proportion of oocytes in which the dye was transferred into more than two inner cumulus cell layers increased. This did not apply to the outermost layer of cumulus cells. Moreover, in this study, at 12-hr cultivation with hormones, a majority of oocytes proceeded from GV II stage to GV III stage and Cx-43 was phosphorylated. The combined evidence from these studies suggests that cultivation with gonadotropins may stimulate the phosphorylation of $\mathrm{Cx}-43$ and then shut down gap junctional communication between cumulus cells in porcine follicular oocytes cultured with hormones, resulting in the induction of the progression from GV II stage to GV III stage.

Cx-43 has numerous phosphorylation sites and these sites are targeted by PKC, pp60 src tyrosine kinase, and MAP kinase (ERK 1,2) [13, 15, 27]. MAP kinase (ERK $1,2)$ phosphorylates three serine sites in $C x-43$ and in- hibits gap junctional communication $[14,15]$. Therefore, we examined the dynamics of activated MAP kinase (ERK 1,2) in porcine cumulus cells by using an antiphospho MAP kinase antibody that reacts to the active form of MAP kinase (ERK 1,2). The electrophoretic mobility of these bands on SDS-PAGE in the present study coincided with active forms of ERK 2 (43 kDa) and ERK 1 (45 kDa) in human endothelial cells [28], mouse 3T3 fibroblast cells [29], and porcine granulosa cells [30]. Although the active form of ERK 1 was detected in all groups, ERK 2 was not found in cumulus cells which were isolated from COCs immediately recovered from their follicles. After 12-hr cultivation in the presence of $\mathrm{FSH}$ and $\mathrm{LH}$, phosphorylated ERK 2 appeared on SDS-PAGE in the $43 \mathrm{kDa}$ region and the unphosphorylated form of $\mathrm{CX}-43$ disappeared. In cases wherein a PI 3-kinase inhibitor, LY294002, was added, the most intense band of the highest migrating form of Cx-43 was concomitant with a distinct band of active ERK 2 with a relatively faint intensity. At the same time point, approximately $60 \%$ of the oocytes in a medium with FSH and LH exhibited the GV III stage. A significantly higher proportion of oocytes were arrested at GV Il stage in the presence of $50 \mu \mathrm{M}$ LY294002. These results suggest that the activation of MAP kinase regulated PI 3-kinase is associated with the progression from GV II stage to GV III stage of oocytes via the control of $\mathrm{Cx}-43$ in cumulus cells.

\section{Acknowledgement}

We are grateful to the staff of the Meat Inspection Office in Hiroshima City for supplying porcine ovaries.

\section{References}

1) Pincus, C and Enzmann, E.V. (1935): The comparative behavior of mammalian eggs in vivo and in vitro. J . Exp. Med., 62, 665-675.

2) Edwards, R.G. (1965): Maturation in vitro of mouse, sheep, cow, pig, rhesus monkey, and human ovarian oocytes. Nature, 23, 349-351.

3) Downs, S.M. and Eppig, J J . (1984): Cyclic adenosine monophosphate and ovarian follicular fluid act synergistically to inhibit mouse oocyte maturation. Endocrinology, 114, 418-427.

4) Downs, S.M., Coleman, D.L. and Eppig, J J . (1986): Maintenance of murine oocyte meiotic arrest: uptake and metabolism of hypoxanthine and adenosine by cumulus cell-endosed and denuded oocytes. Dev. Biol., 117, 174- 183.

5) I sobe, N., Fujihara, M. and Terada, T. (1996): Cumulus cells suppress meiotic progression in pig oocytes 
cultured in vitro. Theriogenology, 45, 1479-1489.

6) Larsen, W.J ., Wert, S.E. and Brunner, G.D. (1986): A dramatic loss of cumulus cell gap junctions is correlated with germinal vesicle breakdown in rat oocytes. Dev. Biol., 113, 517-521.

7) Larsen, W.J ., Wert, S.E. and Brunner, G.D. (1987): Differential modulation of rat follicle cell gap junction populations at ovulation. Dev. Biol., 122, 61-71.

8) Isobe, N., Maeda, T. and Terada, T. (1998): Involvement of meiotic resumption in the disruption of gap junctions between cumulus cells attached to pig oocytes. J . Reprod. Fertil. 113, 167-172.

9) Grazul-Bilska, A.T., Reynolds, L.P. and Redmer, D.A. Gap junctions in the ovaries. Biol. Reprod., 57, 947957.

10) Itahana, K., Morikazu, Y. and Takeya, T. (1996): Differential expression of four connexin genes, Cx-26, $C x-30.3, C x-32$ and $C x-43$, in the porcine ovarian follicle. Endocrinology, 137, 5036-5044.

11) Itahana, K., Tanaka, T., Morikazu, Y., Komatu, S., I shida, N. and Takeya, T. (1998): I solation and characterization of a novel connexin gene, $\mathrm{Cx}-60$, in the porcine ovarian follicles. Endocrinology, 139, 320-329.

12) Masil, L.S., Cunningham, B.A., Edelman, G.M. and Goodenough, D.A. (1990): Differential phosphorylation of the gap junction protein connexin 43 in junctional communication-competent and -deficient cell lines. J . Cell Biol., 111, 2077-2088.

13) Lau, A.F., Kurata, W.E., Kanemitsu, M.Y., Loo, L.W.M., Warn-Cramer, B.J ., Eckhart, W. and Lampe, P.D. (1996): Regulation of connexin 43 function by activated tyrosine protein kinases. J. Bioenerg. Biomembr., 28, 359-367.

14) Hii, C.S.T., Oh, S.Y., Schmidt, S.A., Clark, K.J . and Murray, A.W. (1994): Lysophosphatidic acid inhibits gap-junctional communication and stimulates phosphorylation of connexin-43 in WB cells: possible involvement of the mitogen-activated protein kinase cascade. Biochem. J ., 303, 475-479.

15) Warn-Cramer, B.J., Lampe, P.D., Kurata, W.E., Kanemitsu, M.Y., Loo, L.W.M., Eckhart, W. and Lau, A.F. (1996): Characterization of the mitogen-activated protein kinase phosphorylation sites on the connexin43 gap junction protein. J. Biol. Chem., 271, 37793786.

16) Shimada, M., Anas, M.K.I. and Terada, T. (1998): Effects of phosphatidylinositol 3-kinase inhibitors, wortmannin and LY294002, on germinal veside breakdown (GVBD) in porcine oocytes. J . Reprod. Dev., 44, 281-288.

17) Hu, Q., Klippel, A., Muslin, A.J ., Fantl, W.J . and Williams, L.T. (1995): Ras-dependent induction of cellular responses by constitutively active phosphatidylinositol-
3 kinase. Science, 268, 100-102.

18) Kyriakis, J.M., App, H., Zhang, X., Banerjee, P., Brautigan, D.L., Rapp, U.R. and Avruch, J . (1992): Raf-1 activates MAP kinase-kinase. Nature, 358, 417421.

19) Petters, R.M. and Reed, M..L. (1991): Addition of taurine or hypotaurine to culture medium improves development of one- and two-cell pig embryos in vitro. Theriogenology, 35, 253.

20) Motlik, J and Fulka, J . (1976): Breakdown of germinal vesicle in pig oocytes in vivo and in vitro. J. Exp. Zool. 198, 155-162.

21) Laemmli, U.K. (1970): Cleavage of structual proteins during the assembly of the heat of bacteriophage T4. Nature, 227, 680-685.

22) Lenhart, J .A., Downey, B.R. and Bagnell, C.A. (1998): Connexin 43 gap junction protein expression during follicular development in the porcine ovary. Biol. Reprod. 58, 583-590.

23) Cranot, I. and Dekel, N. (1994): Phosphorylation and expression of connexin-43 ovarian gap junction protein are regulated by luteinizing hormone. J. Biol. Chem., 269, 30502-30509.

24) Wang, Y. and Rose, B. (1997): An inhibition of gapjunctional communication by cadherins. J . Cell Sci., 110, 301-309.

25) Crow, D.S., Beyer, E.C., Paul, D.L., Kobe, S.S. and Lau, A.F. (1990): Phosphorylation of connexin 43 gap junction protein in uninfected and rous sarcoma virus-transformed mammalian fibroblasts. Mol. Cell Biol., 10, 1754-1763.

26) Eppig, J J . (1982): The relationship between cumulus-oocyte coupling, oocyte meiotic maturation, and cumulus expansion. Dev. Biol., 89, 268-272.

27) Loo, L.W.M., Berestecky, J .M., Kanemitsu, M.Y. and Lau, A.F. (1995): pp60src-mediated phosphorylation of connexin 43, a gap junction protein. J . Biol. Chem., 270, 12751-12761.

28) Sexl, V., Mancusi, G., Holler, C., Gloria-Maercker, E., Schutz, W. and Freissmuth, M. (1997): Stimulation of the mitogen-activated protein kinase via the $A_{2 A}$-adenosine receptor in primary human endothelial cells. J. Biol. Chem., 272, 5792-5799.

29) Petrisch, C., Woscholski, R., Edelmann, H.M.L. and Ballou, L.M. (1995): Activation of p70 S6 kinase and erk-encoded mitogen-activated protein kinases is resistant to high cyclic nucleotide levels in swiss 3T3 fibroblasts. J. Biol. Chem., 270, 26619-26625.

30) Cameron, M.R., Foster, J.S., Bukovsky, A. and Wimalasena, J . (1996): Activation of mitogen-activated protein kinase by gonadotropins and cyclic adenosine 5'-monophosphates in porcine granulosa cells. Biol. Reprod., 55, 111-119. 